\title{
Mn(II) Binding by the Anthracis Repressor from Bacillus anthracis ${ }^{\dagger}$
}

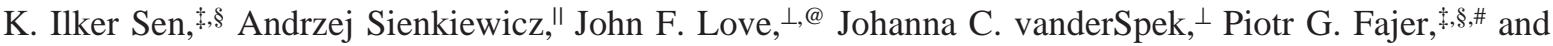 \\ Timothy M. Logan*,\$,,+ \\ Institute of Molecular Biophysics, Florida State University, Tallahassee, Florida 32306, Institute of Physics, Polish Academy of \\ Sciences, Al. Lotników 32/46, 02-668 Warsaw, Poland, Department of Microbiology and Section of Molecular Medicine, \\ Department of Medicine, Boston University School of Medicine, 650 Albany Street, EBRC 830, Boston, Massachusetts 02118, \\ Department of Biological Sciences, Florida State University, Tallahassee, Florida 32306, Department of Chemistry and \\ Biochemistry, Florida State University, Tallahassee, Florida 32306, and National High Magnetic Field Laboratory, \\ 1800 East Paul Dirac Drive, Tallahassee, Florida 32310
}

Received November 8, 2005; Revised Manuscript Received January 24, 2006

\begin{abstract}
The anthracis repressor (AntR) is a manganese-activated transcriptional regulator from Bacillus anthracis and is a member of the diphtheria toxin repressor (DtxR) family of proteins. In this paper, we characterize the $\mathrm{Mn}$ (II) binding and protein dimerization state using a combination of continuous wave (cw) and pulsed EPR methods. Equilibrium metal binding experiments showed that AntR binds 2 equivalents of $\mathrm{Mn}$ (II) with positive cooperativity and apparent dissociation constants of 210 and 16.6 $\mu \mathrm{M}$. AntR showed sub-millisecond Mn(II) on-rates as measured using stopped-flow EPR. The kinetics of $\mathrm{Mn}$ (II) dissociation, measured by displacement with $\mathrm{Zn}(\mathrm{II})$, was biphasic with rate constants of 35.7 and $0.115 \mathrm{~s}^{-1}$. Variable-temperature parallel and perpendicular mode $\mathrm{cw}$ EPR spectra showed no evidence of a spin-exchange interaction, suggesting that the two $\mathrm{Mn}$ (II) ions are not forming a binuclear cluster. Finally, size exclusion chromatography and double electron-electron resonance EPR demonstrated that AntR forms a dimer in the absence of $\mathrm{Mn}(\mathrm{II})$. These results provide insights into the metal activation of AntR and allow a comparison with related DtxR proteins.
\end{abstract}

Bacteria, like all organisms, regulate the amount of transition metal ions in the cell. Of these metals, iron is particularly important as it is essential for viability, but accumulation of iron, especially in the ferrous [Fe(II)] form, results in oxidative damage as the metal is oxidized to the ferric form. Bacteria generally do not accumulate iron or other transition metals as seen in eukaryotic cells and, therefore, require efficient mechanisms for regulating the intracellular concentration of transition metal ions (1). Iron homeostasis is regulated in many bacteria by proteins of the diphtheria toxin repressor (DtxR) ${ }^{1}$ family. These proteins function as metal ion sensors and regulate the transcription of genes involved in metal uptake, storage, and utilization.

$\dagger$ Supported by grants from American Heart Association, FloridaPuerto Rico Affiliate (Grant 0225339B to K.I.S.), National Institutes of Health Grant R01 AI021628 to J. R. Murphy (Boston University) and T.M.L., the Florida State University Research Foundation (T.M.L. and P.G.F.), National Science Foundation Grant MCB 0346650 to P.G.F., and Grant G1MA-CI-2002-4017 (CEPHEUS) of the European Commission (A.S.).

* To whom correspondence should be addressed: Institute of Molecular Biophysics, Kasha Laboratory, Florida State University, Tallahassee, FL 32306-4380. Telephone: (850) 644-8979. Fax: (850) 644-7244. E-mail: logan@sb.fsu.edu.

Institute of Molecular Biophysics, Florida State University.

$\S$ National High Magnetic Field Laboratory.

"Polish Academy of Sciences.

${ }^{\perp}$ Department of Microbiology, Boston University School of Medicine.

@ Section of Molecular Medicine, Department of Medicine, Boston University School of Medicine.

\# Department of Biological Sciences, Florida State University.

${ }^{+}$Department of Chemistry and Biochemistry, Florida State University.
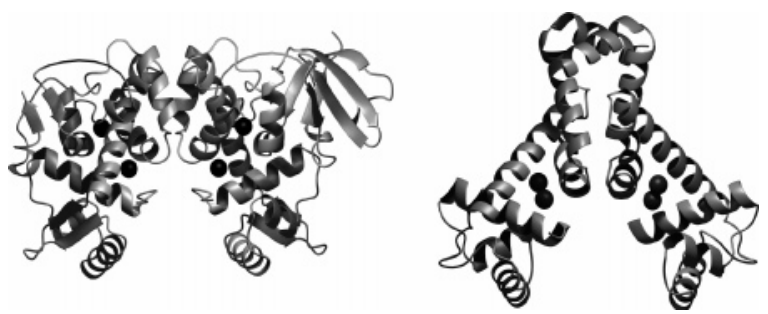

FIGURE 1: Structure of the diphtheria toxin repressor (DtxR, PDB entry $1 \mathrm{c} 0 \mathrm{w}$, left) and manganese transport regulator (MntR, PDB entry 10n1, right). The metals in each protein are represented as dark spheres. The SH3-like domain of the left-side monomer of DtxR is not pictured due to weak electron density. The proteins show strong structural homology in the metal- and DNA-binding domains.

DtxR, the prototypical member of this family of repressors, regulates more than 40 genes in Corynebacterium diphtheriae, and IdeR, from Mycobacterium tuberculosis, regulates approximately 45 different genes (2). In many cases, these repressors have been co-opted to regulate the virulence response $(1,3)$. In $C$. diphtheriae, toxin gene expression is repressed by the metal-activated form of DtxR when the intracellular Fe(II) levels are above a certain threshold. A drop in the Fe(II) level inactivates the repressor and allows active gene transcription.

DtxR proteins typically contain two domains connected by a flexible tether (Figure 1). The larger of the two domains

\footnotetext{
${ }^{1}$ Abbreviations: AntR, Mn(II)-activated repressor from B. anthracis; DtxR, diphtheria toxin repressor; IdeR, iron-dependent repressor from M. tuberculosis; MntR, Mn(II)-activated repressor from B. subtilis.
} 
(N domain) contains a helix - turn-helix DNA binding motif, a dimerization interface, and two structurally and functionally distinct metal binding sites. This domain resembles a molten globule in the apo state (4) but becomes ordered upon metal binding. The smaller C-terminal domain is structurally homologous to eukaryotic SH3 domains. In the holo repressor, this domain contributes two residues to coordinate the ancillary metal binding site (5). In the apo repressor, this domain forms an intramolecular complex with a proline-rich peptide segment that serves to stabilize the inactive repressor (6).

There are a small number of repressors in the DtxR family that contain a single domain that is homologous to the $\mathrm{N}$ domain of DtxR. In these proteins, which include TroR from Treponema pallidum (7), MntR from Bacillus subtilis $(8,9)$, MDR1 from Archeolobus fulgidis (10), and AntR from Bacillus anthracis (11), the DNA binding motif and the residues forming the two metal ion binding sites are strongly conserved with the two-domain members of the DtxR family, but the C-terminal part of the protein is truncated, lacking the proline-rich segment and the SH3-like domain. The crystal structure of MntR (12) established structural homology between MntR and the N-terminal metal- and DNAbinding domain of DtxR (Figure 1). The residues at the C-terminus of MntR contribute to a dimer interface larger than that seen in DtxR and IdeR. Interestingly, the Mn(II) ions in MntR are separated by $\sim 3.3 \AA$, forming a binuclear $\mathrm{Mn}$ (II) cluster. Thus, the metal coordination in MntR is different from that in DtxR and other two-domain members of this family.

It is surprising that metal binding in these proteins is so different, given the level of sequence homology of the metal coordinating ligands. Furthermore, the extended dimer interface and the absence of the SH3 domain suggest that the regulation of repressor activity may also differ from that of DtxR. Here, we investigate Mn(II) binding and dimerization in AntR using a variety of EPR methods. In addition to providing information regarding metal binding by AntR, the results of this study provide insights into metal activation of other members of the DtxR family.

\section{MATERIALS AND METHODS}

Protein Expression and Purification. The AntR cDNA was obtained as described previously (11) and subcloned into the pET11b (New England Biolabs Inc.) expression vector. Direct DNA sequencing of the plasmid confirmed the incorporation of the AntR cDNA in the proper orientation. Recombinant protein was expressed in BL21-CodonPlus(DE3)-RIL competent Escherichia coli cells (Stratagene) in terrific broth medium containing $\mathrm{MgSO}_{4}$ (1 mM), "NPS" [25 mM $\left(\mathrm{NH}_{4}\right)_{2} \mathrm{SO}_{4}, 50 \mathrm{mM} \mathrm{KH}{ }_{2} \mathrm{PO}_{4}$, and $50 \mathrm{mM} \mathrm{Na}_{2} \mathrm{HPO}_{4}$, and " 5052 " (0.5\% glycerol, $0.05 \%$ glucose, and $0.2 \%$ $\alpha$-lactose) to promote self-induction (13). Cells were grown at $37{ }^{\circ} \mathrm{C}$ for $20-24 \mathrm{~h}$, harvested by centrifugation, resuspended in $50 \mathrm{mM}$ Tris ( $\mathrm{pH} 8.5$ ), and disrupted using a highpressure microfluidizer (Microfluidics Co.). The supernatant was clarified by centrifugation, and the protein was purified by anion-exchange chromatography (HiPrep 16/10 Q FF, Amersham Biosciences). AntR eluted at 450-500 mM NaCl.

Fractions containing AntR were identified via SDSPAGE, incubated with $3 \mathrm{M}$ urea and $20 \mathrm{mM}$ EDTA to remove bound metals, and dialyzed exhaustively against Chelex (Bio-Rad Inc.) treated $10 \mathrm{mM}$ HEPES buffer containing $500 \mathrm{mM} \mathrm{NaCl}$ (pH 7.0) (protein buffer) in acidwashed glassware. Trace EDTA was removed by passing samples over a desalting column (HiPrep 26/10 Desalting, Amersham Biosciences). Samples were concentrated to 500 $\mu \mathrm{M}$, with EDTA prewashed ultracentrifugation membranes. The protein concentration was measured in $6 \mathrm{M}$ guanidine hydrochloride from the UV absorbance at $276 \mathrm{~nm}$, using a predicted extinction coefficient of $18450 \mathrm{M}^{-1} \mathrm{~cm}^{-1}$ (14). The metal content of purified AntR was quantified by inductively coupled plasma mass spectrometry (ICP-MS). The total metal ion concentration in a $500 \mu \mathrm{M}$ protein solution was $0.1 \mu \mathrm{M}$ and in Chelex-treated buffer was 0.03 $\mu \mathrm{M}$. The largest contaminant was zinc with concentrations of $\sim 0.09$ and $\sim 0.012 \mu \mathrm{M}$ for protein and buffer, respectively.

The single-cysteine mutant, L15C, was created by use of the QuikChange kit (Stratagene) with primers 5'-TTATATTGAACAAATTTATTGTTTGATTGATGAAAA and 5'-TTTTCATCAATCAAACAATAAATTTGTTCAATATAA purchased from Integrated DNA Technologies, Inc. The mutation was confirmed by direct sequencing. Expression and purification of L15C-AntR were the same as those of the native protein, except that $20 \mathrm{mM}$ dithiothreitol was added to the buffer after the anion-exchange column, and the protein was incubated at $37{ }^{\circ} \mathrm{C}$ for $30 \mathrm{~min}$ to reduce adventitious disulfide bonds before dialysis into demetalated and degassed protein buffer. Purified L15C-AntR $(\sim 100 \mu \mathrm{M})$ was spin-labeled with methanethiosulfonate spin-label (MTSSL) overnight using a 4-fold molar excess of the label. Unreacted label was removed by dialysis against protein buffer.

Native and mutant AntR activity was verified by use of an electrophoretic mobility shift assay (Figure S1 of the Supporting Information). BAmntHO (5'-CCGGTACCTATAATTTGCACTAAGGAAACAGAGCTCTTTACACC$\left.3^{\prime}\right)$ was used as a target DNA sequence (11). DNA (200 nM) and AntR $(20 \mu \mathrm{M})$ were loaded on a $6 \%$ polyacrylamide gel in $40 \mathrm{mM}$ Tris-borate buffer (pH 7.0); $200 \mu \mathrm{M} \mathrm{MnCl}$ was added to the sample, gel, and running buffer immediately prior to running. Gels were stained with SYBR-Gold fluorescent DNA staining agent and imaged using a Typhoon 9200 imager (Amersham Biosciences).

Gel Filtration Chromatography. A Superdex 75 column (Amersham Biosciences) was washed with EDTA and calibrated with a low-molecular weight gel filtration calibration kit (Amersham Biosciences). Six samples of AntR were prepared with $10 \mu \mathrm{M}$ protein and $0-500 \mathrm{mM} \mathrm{NaCl} ; 50 \mu \mathrm{L}$ of each sample was loaded on the column which was equilibrated with the same buffer as the sample. The estimated error in molecular weights of the standards was $10-25 \%$ according to the manufacturer.

Conventional EPR Spectroscopy of $M n(I I) . \mathrm{MnCl}_{2}$ was purchased as a $1.00 \pm 0.01 \mathrm{M}$ solution with $<1 \mathrm{ppm}$ impurities, and $\mathrm{ZnSO}_{4}$ was prepared from heptahydrate powder (both from Sigma-Aldrich, St. Louis, MO). All stock solutions were prepared daily in Chelex-treated, degassed protein buffer. For room-temperature equilibrium binding experiments, AntR was titrated with $\mathrm{MnCl}_{2}(364 \mu \mathrm{M}$ AntR and $1.3 \mathrm{mM} \mathrm{MnCl}_{2}$ in the case of Figure 2; $71 \mu \mathrm{M}$ AntR and 4-20 $\mu \mathrm{L}$ increments of $\mathrm{MnCl}_{2}$ as in Figure 3), and continuous wave (cw) EPR spectra were recorded within 


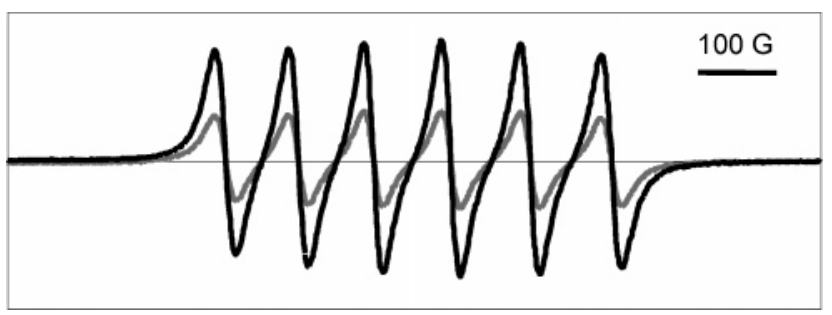

FIGURE 2: EPR spectra of $\mathrm{Mn}(\mathrm{II})$ in the absence (dark line) and presence (light line) of AntR at room temperature in solution. The total $\mathrm{Mn}$ (II) concentration was the same in both samples. The signal intensity decrease corresponds to twice the AntR concentration and, hence, shows AntR binding to two Mn(II) ions.
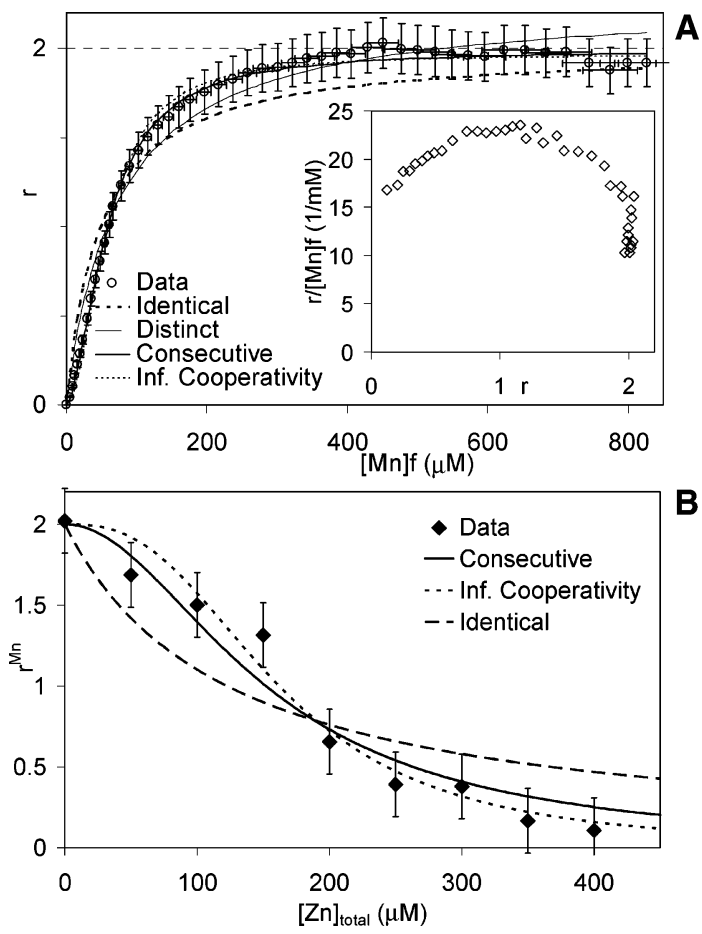

FIGURE 3: Equilibrium binding of manganese and zinc to AntR. (A) The fraction of $\mathrm{Mn}$ (II) bound to AntR ( $r$ ) is calculated from the signal intensity of the lowest-field transition and plotted against the free $\mathrm{Mn}$ (II) concentration. Individual data points represent the average of three independent determinations with one standard deviation indicated by the error bars. The data were fit to specific binding models as described in the text. In the inset is a Scatchard representation of the same data. (B) Fraction of bound Mn(II) with increasing $\mathrm{Zn}(\mathrm{II})$ concentrations and results of fits.

5-10 min of mixing using a Bruker EMX spectrometer (Bruker Biospin, Billerica, MA) with a $\mathrm{TE}_{102}$ cavity in 50 $\mu \mathrm{L}$ glass capillaries (VWR). Competition with $\mathrm{Zn}$ (II) was observed by titrating $0-400 \mu \mathrm{M} \mathrm{Zn}$ (II) into a sample containing $18.5 \mu \mathrm{M}$ AntR and $500 \mu \mathrm{M} \mathrm{Mn}(\mathrm{II})$. Spectra were recorded at $9.6 \mathrm{GHz}$, using a microwave power of $10 \mathrm{~mW}$, a modulation amplitude of $10 \mathrm{G}$, and a modulation frequency of $100 \mathrm{kHz}$. Low-temperature $\mathrm{cw}$ spectra were recorded with a Bruker Elexys 680 spectrometer equipped with a Bruker dielectric ring resonator (model ER 4117D-M).

$\left[\left(\mathrm{Me}_{3} \mathrm{TACN}\right)_{2} \mathrm{Mn}(\mathrm{II})(\mathrm{l}-\mathrm{OAc})_{3}\right] \mathrm{BPh}_{4}$, a model compound containing a $\mathrm{Mn}$ (II) $-\mathrm{Mn}$ (II) binuclear cluster, was a gift of K. Wieghardt (Mülheimer Max-Planck-Institute, Mülheim an der Ruhr, Germany). The powder was dissolved in a dry, oxygen-free 50:50 $\mathrm{CH}_{3} \mathrm{CN} / \mathrm{DMF}$ mixture and transferred to quartz EPR tubes in an argon-filled glovebox, and the tube was flame-sealed. We reproduced the parallel and perpendicular mode spectra of this compound as reported in ref 22 for comparison with our data (Figure S2 of the Supporting Information).

Protein samples for all low-temperature experiments were exchanged into protein buffer containing 30\% ethylene glycol and placed in $4 \mathrm{~mm}$ quartz tubes. These samples contained a 1:1.85 ratio of AntR to $\mathrm{Mn}(\mathrm{II})$, and the absence of free $\mathrm{Mn}(\mathrm{II})$ was confirmed by room-temperature $\mathrm{cw}$ experiments. The spectra were recorded at specific temperatures between 4.2 and $77 \mathrm{~K}$ with a microwave power of $0.02-0.2 \mathrm{~mW}$, a modulation amplitude of $5 \mathrm{G}$, and a modulation frequency of $10 \mathrm{kHz}$. Parallel mode EPR spectra were recorded at the National Biomedical EPR Center (Medical College of Wisconsin, Milwaukee, WI) by B. Bennett. Spectral analysis, including fitting of binding curves, was performed with home-written Labview and MATLAB programs that utilize Monte Carlo-Simplex optimization (15).

Stopped-Flow EPR. The stopped-flow interface with the Bruker EMX spectrometer consisted of a model 1100 syringe ram and a model 715 syringe controller (Update Instruments, Inc., Madison, WI). A home-built dielectric resonator (16) was affixed with the Wiskind mixer (a T-jet mixer followed by a grid mixer; also from Update Instruments). The dead volume of the assembly was estimated to be $\sim 3 \mu \mathrm{L}$. One syringe was loaded with a $\mathrm{Mn}$ (II)-saturated AntR solution $\left(175 \mu \mathrm{M}\right.$ AntR and $750 \mu \mathrm{M} \mathrm{MnCl}_{2}$ ) in protein buffer and the other with $\mathrm{ZnSO}_{4}(750$ or $1500 \mu \mathrm{M})$ freshly prepared in the same buffer. For each transient, a $60 \mu \mathrm{L}$ volume of each syringe was injected into the mixer at $600 \mu \mathrm{L} / \mathrm{s}$ using $6 \mathrm{~mm}$ inside diameter glass syringes. To minimize spectral artifacts arising from mechanical instabilities in the mixing chamber, all the original rubber O-rings attached to the syringe pistons were replaced with custom-made Teflon seals. The magnetic field was fixed at the lowest field $M n$ (II) transition, and kinetic transients were recorded during and after the flow with a microwave power of $2 \mathrm{~mW}$, a microwave frequency of $9.52 \mathrm{GHz}$, a modulation amplitude of $10 \mathrm{G}$, a modulation frequency of $100 \mathrm{kHz}$, a time constant of $5.12 \mathrm{~ms}$, a conversion time of $5.12 \mathrm{~ms}$, a static magnetic field of 3145 $\mathrm{G}$, and 8192 acquisition points. At the end of each kinetic experiment, cw EPR spectra of the AntR-Mn-Zn species were collected 30, 60, and 120 min after the mixing to ensure equilibration.

Pulsed EPR. Inter-spin-label distance measurements were performed on a Bruker Elexys 680 spectrometer using the four-pulse double electron-electron resonance (DEER) experiment (17). DEER samples included $300 \mu \mathrm{M}$ singly labeled AntR in protein buffer containing 30\% ethylene glycol. The static field was set to the low-field resonance of the nitroxide signal. The frequency of the pumping microwave was matched to the resonator frequency, while the frequency of microwave observation pulses was adjusted to be $65 \mathrm{MHz}$ higher than the pump frequency. The $\pi / 2$ pulse length was $16 \mathrm{~ns}$, and the echo above the noise level was integrated. All data manipulations were accomplished with software developed by G. Jeschke (available at http:// www.mpip-mainz.mpg.de/ jeschke/). The resulting distance populations are based on hermite interpolation.

\section{RESULTS}

Oligomerization State. AntR exhibited extensive aggregation in HEPES buffer which was controlled by $\mathrm{NaCl}$. To 


\begin{tabular}{|c|c|c|c|c|c|}
\hline \multicolumn{2}{|r|}{ model } & \multicolumn{2}{|c|}{ Mn(II) binding } & \multicolumn{2}{|c|}{$\mathrm{Zn}(\mathrm{II})$ binding } \\
\hline name & fraction bound & $K_{\mathrm{D}}(\mu \mathrm{M})$ & $\chi^{2}$ & $K_{\mathrm{D}}(\mu \mathrm{M})$ & $\chi^{2}$ \\
\hline identical & $r=\frac{2[\mathrm{Mn}]_{\mathrm{f}}}{K_{\mathrm{D}}+[\mathrm{Mn}]_{\mathrm{f}}}$ & $49 \pm 10$ & 1.35 & $123 \pm 139$ & 0.78 \\
\hline distinct & $r=\frac{n_{1}[\mathrm{Mn}]_{\mathrm{f}}}{K_{\mathrm{D} 1}+[\mathrm{Mn}]_{\mathrm{f}}}+\frac{n_{2}[\mathrm{Mn}]_{\mathrm{f}}}{K_{\mathrm{D} 2}+[\mathrm{Mn}]_{\mathrm{f}}}$ & $\begin{array}{l}73 \pm 20 \\
73 \pm 20 \\
n_{1}+n_{2}=2.3\end{array}$ & 0.55 & $\begin{array}{l}123 \pm 139 \\
123 \pm 139 \\
\left(n_{1}+n_{2}=2.0\right)\end{array}$ & 0.78 \\
\hline consecutive & $r=\frac{K_{\mathrm{D} 2}[\mathrm{Mn}]_{\mathrm{f}}}{K_{\mathrm{D} 1} K_{\mathrm{D} 2}+K_{\mathrm{D} 2}[\mathrm{Mn}]_{\mathrm{f}}+[\mathrm{Mn}]_{\mathrm{f}}^{2}}$ & $\begin{array}{c}210 \pm 18 \\
16.6 \pm 1.0\end{array}$ & 0.05 & $\begin{array}{l}5 \times 10^{7} \pm 1 \times 10^{12} \\
4 \times 10^{-4} \pm 9\end{array}$ & 0.19 \\
\hline infinite cooperativity & $r=\frac{2[\mathrm{Mn}]_{\mathrm{f}}^{2}}{K_{\mathrm{D}}^{2}+[\mathrm{Mn}]_{\mathrm{f}}^{2}}$ & $53.9 \pm 18$ & 0.15 & $152 \pm 47$ & 0.16 \\
\hline
\end{tabular}

characterize AntR's oligomerization state, the effective hydrodynamic radius of AntR was determined as a function of $\mathrm{NaCl}$ concentration using size exclusion chromatography. At $\leq 100 \mathrm{mM}$ salt, the entire AntR sample eluted in the void volume, suggesting the formation of high-molecular mass aggregates. The amount of protein appearing in the void volume was reduced to $\sim 5 \%$ of the total amount of protein as the salt concentration increased to $200 \mathrm{mM}$ and disappeared at higher concentrations. The hydrodynamic radius of apo-AntR $(10 \mu \mathrm{M})$ corresponded to that of a $34.5 \pm 4.4 \mathrm{kDa}$ globular protein at $500 \mathrm{mM} \mathrm{NaCl}$. This is in agreement with dimerized AntR, which has a computed molecular mass of $33.2 \mathrm{kDa}$. AntR exhibited similar molecular masses $(34.6 \pm 4.0$ and $34.2 \pm 3.6 \mathrm{kDa})$ in buffers containing 400 and $300 \mathrm{mM} \mathrm{NaCl}$. Considering that our biophysical experiments would be performed at higher protein concentrations, which may positively contribute to aggregation at low salt concentrations, we used $500 \mathrm{mM}$ $\mathrm{NaCl}$ in the protein buffer.

Binding Constant and Stoichiometry. $\mathrm{Mn}(\mathrm{II})$ is a paramagnetic ion for which $S=5 / 2$ and $I=5 / 2$. Spectra of aqueous $\mathrm{Mn}(\mathrm{II})$ at room temperature typically exhibit six lines centered at $g \approx 2$ arising from the hyperfine interaction of manganese electrons with ${ }^{55} \mathrm{Mn}$ nuclei. When $\mathrm{Mn}$ (II) is bound to a protein, its room-temperature X-band signal disappears (Figure 2) due to a broadening via the zero-field splitting arising from the disturbances in the octahedral ligand field of the bound metal (18). Thus, Mn(II) resonances observed in room-temperature solution EPR spectra in the presence of varying concentrations of AntR arise from only unbound $\mathrm{Mn}$ (II) ions free in solution, and the amplitude of the $\mathrm{Mn}$ (II) signal can be used to determine binding energetics.

High concentrations of $\mathrm{NaCl}$ suppress the $\mathrm{Mn}(\mathrm{II}) \mathrm{EPR}$ signal intensity to a small extent, possibly due to replacement of water in the coordination sphere with $\mathrm{Cl}^{-}$ions, which reduces the symmetry of the ligand field. The salt-induced change in $\mathrm{Mn}$ (II) resonance intensity does not affect the binding parameters obtained in our experiments as all spectra were collected at a constant salt concentration and normalized to the $\mathrm{Mn}$ (II) resonance intensity in the absence of AntR.

The binding stoichiometry was calculated directly from the decrease in the magnitude of the free manganese signal upon addition of a fixed amount of AntR (Figure 2) and corresponded to two $\mathrm{Mn}$ (II) ions bound per AntR molecule.
The entire titration curve (Figure 3) exhibits a weak sigmoidal character. Quantitative estimates of binding parameters were obtained from curve fitting using the following binding models: (1) two independent sites with identical dissociation constants, (2) two independent sites with individual dissociation constants and stoichiometries, (3) two sites exhibiting a finite positive cooperativity, and (4) two sites with infinite cooperativity, e.g., both ligands binding simultaneously and no singly ligated species. Equations for the fraction of bound $\mathrm{Mn}$ (II) versus free $\mathrm{Mn}$ (II) in solution, the resulting apparent dissociation constants, and $\chi^{2}$ for the fits to the above models are given in Table 1. Statistical analysis between infinite cooperativity (simpler model) and the consecutive binding model yielded an $F$-test result of 33.7 and a $P$ value of $<5 \%(19,20)$. Thus, model 3 (consecutive binding with positive cooperativity) yields the best result, with apparent $K_{\mathrm{D}}$ 's equal to $210 \pm 18$ and $16.6 \pm 1.0 \mu \mathrm{M}$ for the two binding sites. Transforming the binding data into a Scatchard representation (Figure 3, inset) indicated a concave-downward plot, confirming the positive cooperative nature of Mn(II) binding by AntR (21).

The apparent affinity of AntR for $\mathrm{Zn}$ (II) was determined by a competitive binding assay. $\mathrm{Zn}$ (II) is a structural analogue of $\mathrm{Mn}(\mathrm{II})$ and activates AntR for DNA binding (11) but is diamagnetic and EPR silent. Zn(II) was titrated into Mn(II)-saturated AntR, and the increase in the Mn(II) signal intensity as a result of replaced ions was monitored using EPR. The resulting curve has a sigmoidal character, and all bound $\mathrm{Mn}(\mathrm{II})$ was completely displaced at a 1:1 Mn(II):Zn(II) ratio (Figure 3 ). The binding curve was modeled as described in Table 1 , but by using $r^{\mathrm{Mn}}=2-r^{\mathrm{Zn}}$, where 2 is the total number of binding sites and $r^{\mathrm{Zn}}$ is the number of bound $\mathrm{Zn}(\mathrm{II})$ ions per AntR. It is assumed that $[\mathrm{Zn}(\mathrm{II})]_{\text {free }} \approx$ $[\mathrm{Zn}(\mathrm{II})]_{\text {total }}$. From the considered models, infinite cooperativity model 4 was statistically the best with an apparent $K_{\mathrm{D}}$ of $152 \pm 47 \mu \mathrm{M}$ and a $\chi^{2}$ of 0.19 .

Binding Environment. Low-temperature cw EPR spectra of $\mathrm{Mn}$ (II)-activated AntR in both the perpendicular and parallel modes are shown in Figure 4. The perpendicular mode spectra are dominated by six transitions centered at $g \sim 2$ with average hyperfine coupling of $9.1 \mathrm{mT}$. Additional signals within this six-line multiplet arise from forbidden transitions $\left(\Delta m_{\mathrm{I}}=1\right)$ which have increased probability at low temperatures. There was no major change in the spectra recorded between 10 and $70 \mathrm{~K}$, nor was there evidence of a 


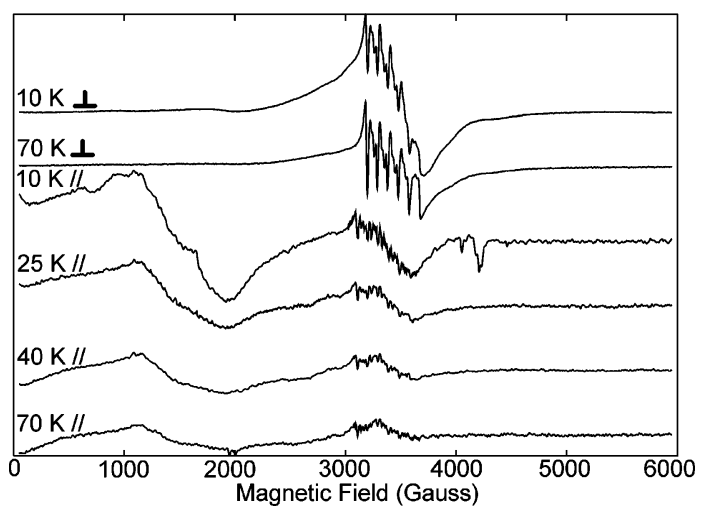

FIGURE 4: Parallel and perpendicular mode EPR spectra of Mn(II)-bound AntR at various temperatures. For these samples, the absence of free $\mathrm{Mn}(\mathrm{II})$ in solution was confirmed by roomtemperature cw EPR on identical samples.

$4.5 \mathrm{mT}$ coupling that would indicate the presence of a spinexchange interaction (Figure S2). Parallel mode spectra of $\mathrm{Mn}$ (II)-loaded AntR (Figure 4) exhibited residual perpendicular mode transitions at $g \sim 2$ and a broad featureless transition centered at $g \sim 4.9$. A similar transition was observed previously and attributed to $S=1$ spins in a dimanganese cluster (22). However, the $S=1$ transitions in parallel mode spectra are expected to increase in intensity between 0 and $\sim 15 \mathrm{~K}$ and decay slowly as the temperature is increased (23), whereas the signal for $\mathrm{Mn}$ (II)-loaded AntR shows a relatively weak temperature dependence.

Kinetics of Metal Binding and Release. The binding kinetics was determined using a rapid mixing apparatus coupled to the EPR resonator (16). Experiments performed to measure the on-rate at saturating $\mathrm{Mn}$ (II) levels showed a flat kinetic trace, indicating that binding went to completion during the dead time of the instrument and yielding a $k_{\text {on }}$ of $>1000 \mathrm{~s}^{-1}$ (not shown). The dissociation kinetics was measured by introducing $\mathrm{Zn}(\mathrm{II})$ and observing the time course for the increase in signal intensity (Figure 5). When $\mathrm{Mn}$ (II)-saturated AntR was mixed with $\mathrm{Zn}$ (II) to a final Mn: $\mathrm{Zn}$ molar ratio of $1: 1$, the intensity increased in a biphasic manner with each phase exhibiting approximately equal amplitudes.

The transient was described by a double exponential:

$$
y=y_{0}-A_{1} \mathrm{e}^{k_{1} t}-A_{2} \mathrm{e}^{-k_{2} t}
$$

yielding apparent kinetic rate constants $k_{1}$ and $k_{2}$ of $35.7 \pm$ 12.1 and $0.115 \pm 0.009 \mathrm{~s}^{-1}$, respectively. A 2-fold increase in $\mathrm{Zn}$ (II) concentration showed very similar rates $(29.7 \pm$ 15.6 and $0.117 \pm 0.004 \mathrm{~s}^{-1}$, respectively), and further increases resulted in protein precipitation. Conventional scanned cw EPR spectra were recorded immediately prior to and 30 min after mixing with $\mathrm{Zn}(\mathrm{II})$, indicating the end points of the kinetic experiment. The absolute signal intensity change in these spectra demonstrated that the two phases in the kinetic trace account quantitatively for the total signal change, signifying the absence of burst or slow kinetic phases for metal exchange.

Dimerization. The dimerization of AntR was investigated by measuring the distance between spin-labeled monomers. Residue L15 was mutated to cysteine and labeled with MTSSL nitroxide spin-label. Spin-labeled L15C-AntR bound
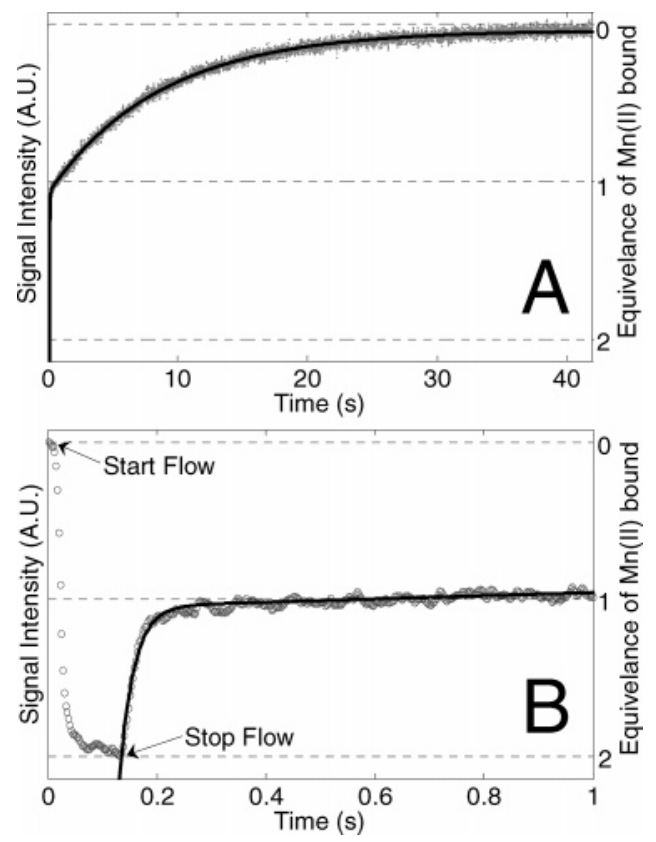

FIGURE 5: Kinetics of dissociation of $\mathrm{Mn}$ (II) from AntR at room temperature. (A) Time dependence of the Mn(II) EPR signal (data points) following mixing of $\mathrm{Mn}(\mathrm{II})$-saturated AntR with an excess of $\mathrm{Zn}(\mathrm{II})$, and the fit (solid line). (B) Expansion of the early time points of the kinetic transient shown in panel A. The signal level at time zero is the equilibration point which will eventually $(t \sim$ $60 \mathrm{~s})$ be attained after mixing. During mixing (15 ms $<t<135$ $\mathrm{ms}$ ), the signal rapidly reaches that of a $\mathrm{Mn}(\mathrm{II})$-bound AntR solution in one of the syringes, and when the flow is stopped $(t=135 \mathrm{~ms})$, the intensity increases due to $\mathrm{Mn}$ (II) being replaced in AntR. The real kinetics of $\mathrm{Mn}$ (II) replacement starts after this point.

to DNA in a manner indistinguishable from that of the wild type (Figure 2S). The interspin distance between the nitroxides was measured by double electron-electron resonance (DEER). In the DEER experiment, dipolar coupling between electron spins is observed as a modulation of the spin-echo intensity due to spin inversion of its dipolar coupled partner. The intensity of the refocused echo is measured as a function of the delay time of the inversion pulse. Fourier transformation of the echo modulation results in a Pake pattern, with singularities being inversely proportional to the cubic distance between spins.

The DEER spectrum obtained for apo L15C-AntR is shown in Figure 6A. The change in echo intensity with respect to delay time $t$ is the modulation of echo overlapped with the background. The background arises from intermolecular dipole-dipole interactions distributed homogeneously in solution. This contribution can be estimated (17) and subtracted, resulting in the pure echo modulation (Figure 6B). The frequency of the modulation (Figure 6C) was deconvoluted into the distance domain showing three populations spanning a $2 \mathrm{~nm}$ range (Figure 6D). The most probable interspin distance is $3.3 \mathrm{~nm}$ with a mean distance of $3.7 \mathrm{~nm}$. In our experience, the distances and populations deconvoluted from high signal-to-noise DEER spectra, such as those reported here, can easily be resolved with this procedure. Despite the fact that each monomer of L15C-AntR is labeled with a single nitroxide spin-label, the DEER spectrum clearly shows echo modulation, which indicates that apo-AntR is oligomeric in solution. 

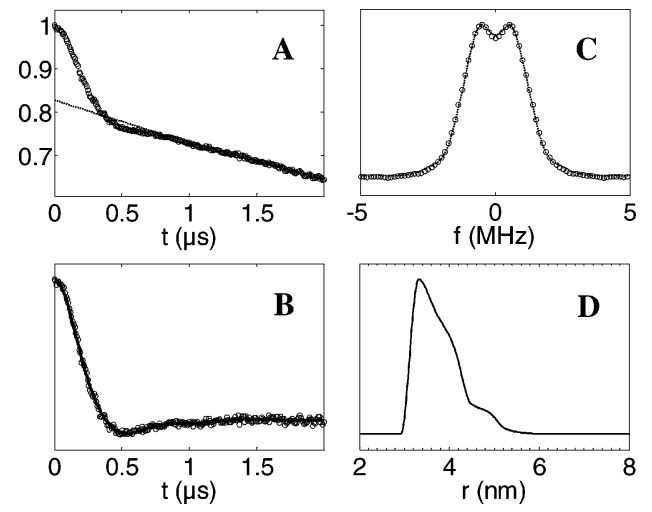

FIGURE 6: DEER spectrum of apo-L15C-AntR MTSSL. (A) Modulation of the echo intensity with varying pump pulse delay time $(\mathrm{O})$ and the decaying background which is due to interdimer interactions $(\cdots)$. (B) Background subtracted data $(O)$ and the deconvolution fit $(-)$. (C) Resulting Pake pattern from Fourier transformation of the data in panel B. (D) Final distance distribution.

\section{DISCUSSION}

The DtxR family can be divided into proteins having one or two domains; DtxR and IdeR contain a C-terminal SH3like domain and belong to the latter category, whereas AntR and its homologue MntR lack this SH3-like domain and belong to the former. The two subfamilies exhibit homology in the amino acid residues participating in metal binding, DNA binding, and dimerization. The SH3-like domain regulates the energetics of metal activation by affecting the dimerization state in the apo repressor ( 6 ) and by coordinating the metal in the ancillary metal binding site in the holo repressor (5). Further evidence for the regulatory nature of the SH3-like domain comes from the single site mutation in the SH3-like domain of DtxR and IdeR that results in a hyperactive repressor (24-26).

The recently determined crystal structure of metal-bound MntR revealed unexpected differences in metal coordination between the single- and two-domain proteins. In DtxR and IdeR, metals are coordinated in two structurally and functionally distinct sites, separated by $\sim 9 \AA$. This coordination is relatively insensitive to the specific metal being bound (27). On the other hand, MntR binds 2 equiv of $\mathrm{Mn}(\mathrm{II})$ in a binuclear cluster containing two bridging carboxylate and one water ligand with an internuclear distance of $3.3 \AA$ (12). Given these differences, investigating metal binding and activation in single-domain members of this family is particularly interesting.

$\mathrm{Mn}(\mathrm{II})$ binding by AntR was characterized using a combination of pulsed and cw EPR methods. AntR binds 2 equiv of $\mathrm{Mn}$ (II) as shown by the equilibrium ligand binding studies, which saturate at 2 equiv of $\mathrm{Mn}$ (II) (Figure 3). The concave-downward Scatchard plot (Figure 3, inset) provides unambiguous evidence of positive cooperative binding of $\mathrm{Mn}$ (II) ions by AntR. A more quantitative estimate of the ligand binding energetics was obtained by fitting the data to a variety of binding models, including independent and interacting sites. Curve fitting using models having noninteracting sites yields high $\chi^{2}$ values and nonrandom residuals. In contrast, fitting the data to models that include interacting sites improves the fit (Figure 3 and Table 1). Despite the fact that both cooperative binding models fit within the error range of the experiment, consecutive binding improves the

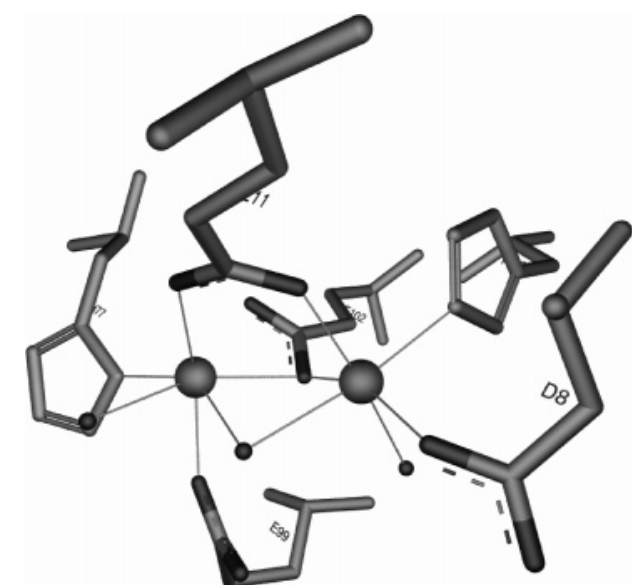

FIGURE 7: Metal binding sites of MntR (12). The bridging atoms and coordination of the two manganese ions are indicated.

fitting significantly, as confirmed by an $F$-test. The binding model based on sequential binding of the ligands with positive cooperativity also provides the more realistic model, yielding apparent equilibrium dissociation constants of $210 \pm 18$ and $16.6 \pm 1.0 \mu \mathrm{M}$ for the low- and high-affinity states, respectively.

Cooperative metal binding has not been directly characterized in the DtxR family of proteins. A recent study of IdeR clearly demonstrated positive cooperativity for metal-linked DNA binding (28). Since IdeR contains two tryptophans and metal-induced fluorescence changes arise not only from metal quenching but also from metal-induced conformational changes, analysis of metal binding cooperativity was complicated. Target DNA binding by metal-activated DtxR and IdeR is cooperative $(28,29)$, as the binding of the first dimer enhances the binding of the second. Cooperative metal binding in AntR may be an evolutionary step compensating for the lack of a SH3-like domain, which has a regulatory role in DtxR (6).

It is striking that DtxR and MntR coordinate metal in fundamentally different ways given the near conservation of the metal coordinating residues between the two proteins. Therefore, we were particularly interested to determine whether AntR coordinates the $\mathrm{Mn}(\mathrm{II})$ ions in a manner resembling that of DtxR or MntR. Here, we used variabletemperature multimode $\mathrm{cw}$ EPR spectroscopy to investigate the hypothesis that spin-exchange coupling exists in the manganese-bound state of AntR. The presence of spinexchange coupling would provide clear evidence that the two $\mathrm{Mn}(\mathrm{II})$ ions are forming a binuclear cluster in AntR, as suggested from the MntR structure (Figure 7). This approach has been used to identify the formation of a binuclear $\mathrm{Mn}$ (II) cluster in aminopeptidases $(30,31)$, concanavalin A (32), phosphatase (33), thiosulfate oxidase (34), arginase (35), phosphotriesterase (36), and enolase (37). As shown in Figure 4, our low-temperature cw EPR spectra lack the spectral signatures associated with spin-exchange coupling and therefore are not consistent with formation of a binuclear manganese cluster in AntR. Spin-exchange coupling is strongly dependent on the metal-metal distance, the chemistry of the bridging atoms, and their coordination geometry (38). The simplest explanation for the absence of spinexchange coupling between the two bound $\mathrm{Mn}$ (II) ions in AntR is that the two metals are separated by more than 
$\sim 4 \AA$. This does not exclude the possibility that the Mn(II) ions are bound within $4 \AA$ of each other but are coordinated such that the spin-exchange coupling is quenched. However, to our knowledge, there are no examples of such $\mathrm{Mn}$ (II)containing proteins or compounds in which the two $\mathrm{Mn}$ (II) ions are separated by $<4 \AA$ and do not exhibit spin-exchange coupling.

All DtxR family proteins function as metal-activated dimeric proteins. DtxR exists as a weakly dimeric protein in the absence of metal $\left(K_{\mathrm{D}} \sim 3 \mu \mathrm{M}\right)$, and metal binding effects a strong shift toward the dimeric state $\left[K_{\mathrm{D}} \sim 33 \mathrm{nM}\right.$ (39)]. Removing the SH3-like domain from DtxR increases dimer affinity in the apo state (6). In contrast, MntR appears to be largely dimeric in the apo state, with little change in dimer affinity upon metal binding (40). Considering the possible structural differences between AntR and MntR based on the equilibrium metal binding and coordination studies presented here, we investigated the dimerization state of AntR in the absence of metal to determine whether AntR was more similar to DtxR or MntR.

AntR more closely resembles MntR in this case as a measurable amount of dimeric protein is present in the absence of metal. The DEER experiment is essentially the EPR correlate of the FRET or NOESY experiment and is a sensitive means of investigating dipolar interactions between electrons. The distance sensed by the DEER experiment falls in the range of $17-70 \AA$. Here, we see a strong modulation of the electron spin-echo envelope in the L15C mutant of AntR. Since there is a single spin-label introduced per monomer of AntR, the modulation must arise from dipolar coupling with another spin-labeled AntR protein, as expected in an oligomeric structure. Size exclusion chromatography results restrict the oligomerization state to a dimer, as the calculated molecular mass of the AntR dimer agrees with the apoprotein's elution profile. The echo modulation was analyzed to yield the interspin distance with a mean distance of $37 \AA$. The distance distribution (Figure 6D) is sufficiently narrow to arise from a single population of dimers, suggesting the formation of a specific dimer interface. The spinlabel side chain has an approximate length of 5-6 $\AA$ and can exhibit motion independent of the protein. We cannot conclude from the DEER spectra whether the width in distance distribution is due to motions inherent in the spinlabel on AntR, multiple conformers of the backbone, or both. However, the intermonomer $\mathrm{C}_{\alpha}-\mathrm{C}_{\alpha}$ distance for the homologous residue (M15) in MntR is $4.1 \mathrm{~nm}$ (12). Therefore, considering the extension of the spin-label side chains, the observed distance between nitroxides is in agreement with this structure.

A recent study of MntR showed a high affinity for dimer in the absence of metal with little further enhancement in dimer formation upon metal binding (40). MDR1, a singledomain DtxR homologue found in archae bacteria, is reported to exist as a dimeric protein in the absence of metal (10). In addition to our observation that apo-AntR is dimeric, there appears to be a general trend that the single-domain members of the DtxR family (AntR and MntR) exhibit a higher affinity for dimer formation in the apo state than the two-domain proteins (DtxR and IdeR) and that the amount of dimer is essentially independent of bound metal. The increased level of dimerization of single-domain members most likely results from the more extensive dimer interface observed for MntR

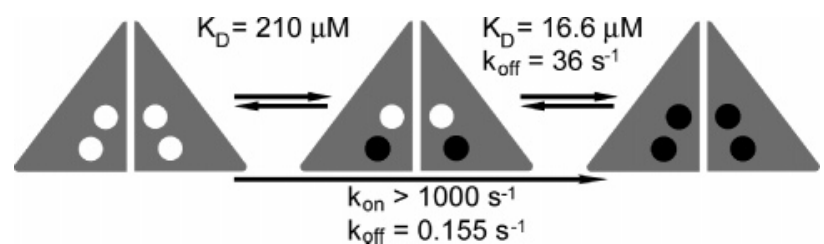

FIGURE 8: Activation mechanism of AntR. White circles represent empty sites, and black circles are $\mathrm{Mn}$ (II)-bound states. These thermodynamic sites are not yet assigned to physical sites within AntR.

than for members of the two-domain subfamily. We predict that a similar dimer interface should exist in the other members of the single-domain family (AntR and MDR1) due to a high degree of sequence homology.

The kinetics of metal binding or dissociation has not been previously reported for any DtxR homologue. The binding rate was faster than the resolution of our instrument $\left(k_{\mathrm{on}}>\right.$ $1000 \mathrm{~s}^{-1}$ ). The dissociation rate was measured by displacement of bound $\mathrm{Mn}(\mathrm{II})$ with $\mathrm{Zn}(\mathrm{II})$. The EPR-silent $\mathrm{Zn}$ (II) is a good analogue of $\mathrm{Mn}(\mathrm{II})$ as the $\mathrm{Zn}$-bound $\mathrm{AntR}$ is capable of recognizing and binding to the cognate AntR promoter sequence (11). Furthermore, equilibrium studies indicated that $\mathrm{Zn}(\mathrm{II})$ can quantitatively replace bound $\mathrm{Mn}(\mathrm{II})$ ions (Figure 3) and the displacement kinetics did not depend on $\mathrm{Zn}$ (II) concentration (over the 2-fold concentration range). Hence, the kinetics is determined by the dissociation of $\mathrm{Mn}$ (II) from AntR and not by the binding of $\mathrm{Zn}$ (II) to the protein . Linking the kinetic rates to equilibrium binding constants at this stage is complicated by the cooperative nature of metal binding and by uncertainty regarding the molecular identity of the intermediate species formed during replacement of $\mathrm{Mn}(\mathrm{II})$ with $\mathrm{Zn}$ (II). These intermediates may include, for instance, AntR species with one site occupied by Zn(II) and the other with $\mathrm{Mn}(\mathrm{II})$, species in which one site is occupied by metal [either $\mathrm{Mn}$ (II) or $\mathrm{Zn}(\mathrm{II})$ ] while the other site is unoccupied, AntR with both sites unoccupied, exchange of metal between sites, etc. We can, however, hypothesize that the binding of a second $\mathrm{Mn}$ (II) with an apparent dissociation constant $\left(K_{\mathrm{D}}\right)$ of $16.6 \mu \mathrm{M}$ exhibits a rapid on $\leftrightarrow$ off equilibrium for which $k_{\mathrm{off}}$ is $\sim 36 \mathrm{~s}^{-1}$ and $k_{\mathrm{on}}$ is faster than $1000 \mathrm{~s}^{-1}$. These results are summarized in Figure 8.

Manganese is required for several metabolic enzymes and, as a cofactor for superoxide dismutase, is involved in the bacterial oxidative stress response (41). Bacteria must be able to accurately sense intracellular manganese concentrations and mount appropriate responses to fluctuations in the steadystate concentration of $\mathrm{Mn}$ (II). E. coli, for instance, maintains 10-100 $\mu \mathrm{M}$ manganese in the cell (42). The intracellular manganese concentration in $B$. anthracis is not known; however, B. subtilis requires at least $80 \mu \mathrm{M} \mathrm{Mn(II)} \mathrm{within}$ the cell for sporulation (43). Eisenstadt et al. (44) have reported that in a controlled culture medium where the added $\mathrm{Mn}(\mathrm{II})$ concentration is less than $1 \mu \mathrm{M}, B$. subtilis accumulates $90 \%$ of the external $\mathrm{Mn}(\mathrm{II})$, and the manganese content of the cell is proportional to the external $\mathrm{Mn}$ (II) concentration. The free Mn(II) concentration during bacterial growth and early sporulation could be as much as $300 \mu \mathrm{M}$. These values for intracellular free manganese are precisely in the range of AntR metal affinity. Our biophysical results indicate that AntR would respond rapidly and linearly to fluctuations in manganese concentration in this range by 
cycling between partially and fully ligated states. Thus, like MntR, AntR may provide the molecular signal transducer that regulates manganese homeostasis in $B$. anthracis.

\section{ACKNOWLEDGMENT}

We thank Brian Bennett for help in acquisition of the parallel mode EPR spectra at the Medical College of Wisconsin. We also thank John Murphy and Bob Harrison for stimulated discussion and sharing their data prior to publication and Vincent Salters for ICP-MS measurements.

\section{SUPPORTING INFORMATION AVAILABLE}

In vitro activation of AntR and perpendicular and parallel mode cw EPR spectra of the model di-Mn compound [ $\left(\mathrm{Me}_{3}-\right.$ $\left.\mathrm{TACN})_{2} \mathrm{Mn}(\mathrm{II})(1-\mathrm{OAc})_{3}\right] \mathrm{BPH}_{4}$. This material is available free of charge via the Internet at http://pubs.acs.org.

\section{REFERENCES}

1. Braun, V. (2001) Iron uptake mechanisms and their regulation in pathogenic bacteria, Int. J. Med. Microbiol. 291, 67-79.

2. Gold, B., Rodriguez, G. M., Marras, S. A. E., Pentecost, M., and Smith, I. (2001) The Mycobacterium tuberculosis IdeR is a dual functional regulator that controls transcription of genes involved in iron acquisition, iron storage and survival in macrophages, $\mathrm{Mol}$. Microbiol. 42, 851-865.

3. Ratledge, C. (2004) Iron, mycobacteria and tuberculosis, Tuberculosis (Edinburgh) 84, 110-130.

4. Twigg, P. D., Parthasarathy, G., Guerrero, L., Logan, T. M., and Caspar, D. L. D. (2001) Disordered to ordered folding in the regulation of diphtheria toxin repressor activity, Proc. Natl. Acad. Sci. U.S.A. 98, 11259-11264.

5. Pohl, E., Holmes, R. K., and Hol, W. G. J. (1999) Crystal structure of a cobalt-activated diphtheria toxin repressor-DNA complex reveals a metal-binding SH3-like domain, J. Mol. Biol. 292, 653667.

6. Wylie, G. P., Rangachari, V., Bienkiewicz, E. A., Marin, V., Bhattacharya, N., Love, J. F., Murphy, J. R., and Logan, T. M. (2005) Prolylpeptide binding by the prokaryotic SH3-like domain of the diphtheria toxin repressor: A regulatory switch, Biochemistry 44, 40-51.

7. Posey, J. E., Hardham, J. M., Norris, S. J., and Gherardini, F. C. (1999) Characterization of a manganese-dependent regulatory protein, TroR, from Treponema pallidum, Proc. Natl. Acad. Sci. U.S.A. 96, 10887-10892.

8. Patzer, S. I., and Hantke, K. (2001) Dual repression by $\mathrm{Fe}^{2+}$-Fur and $\mathrm{Mn}^{2+}-\mathrm{MntR}$ of the mntH gene, encoding an NRAMP-like $\mathrm{Mn}^{2+}$ transporter in Escherichia coli, J. Bacteriol. 183, 48064813.

9. Que, Q., and Helmann, J. D. (2000) Manganese homeostasis in Bacillus subtilis is regulated by MntR, a bifunctional regulator related to the diphtheria toxin repressor family of proteins, Mol. Microbiol. 35, 1454-1468.

10. Bell, S. D., Cairns, S. S., Robson, R. L., and Jackson, S. P. (1999) Transcriptional regulation of an archaeal operon in vivo and in vitro, Mol. Cell 4, 971-982.

11. Love, J. F., Savla, A., Harrison, R., vanderSpek, J. C., and Murphy, J. R. (2006) AntR, a manganese-dependent DtxR homologue from Bacillus anthracis: Initial characterization of operator sequence requirements, manuscript submitted for publication.

12. Glasfeld, A., Guedon, E., Helmann, J. D., and Brennan, R. G. (2003) Structure of the manganese-bound manganese transport regulator of Bacillus subtilis, Nat. Struct. Biol. 10, 652-657.

13. Grabski, A., Mehler, M., and Drott, D. (2003) Unattended highdensity cell growth and induction of protein expression with the Overnight Express ${ }^{\mathrm{TM}}$ Autoinduction System, InNovations 17, 3-6.

14. Gasteiger, E., Gattiker, A., Hoogland, C., Ivanyi, I., Appel, R. D., and Bairoch, A. (2003) ExPASy: The proteomics server for in-depth protein knowledge and analysis, Nucleic Acids Res. 31, 3784-3788.

15. Fajer, P. G., Bennett, R. L. H., Polnaszek, C. F., Fajer, E. A., and Thomas, D. D. (1990) General-Method for Multiparameter Fitting of High-Resolution Epr-Spectra Using a Simplex Algorithm, $J$. Magn. Reson. 88, 111-125.

16. Sienkiewicz, A., Ferreira, A. M. D., Danner, B., and Scholes, C. P. (1999) Dielectric resonator-based flow and stopped-flow EPR with rapid field scanning: A methodology for increasing kinetic information, J. Magn. Reson. 136, 137-142.

17. Pannier, M., Veit, S., Godt, A., Jeschke, G., and Spiess, H. W. (2000) Dead-time free measurement of dipole-dipole interactions between electron spins, J. Magn. Reson. 142, 331-340.

18. Reed, G. H., and Cohn, M. (1970) Electron Paramagnetic Resonance Spectra of Manganese(II)-Protein Complexes. Manganese(II)-Concanavalin-A, J. Biol. Chem. 245, 662.

19. Gonick, L., and Smith, W. (1993) The Cartoon guide to Statistics, Harper Perennial, New York.

20. Hoel, P. G., Port, S. C., and Stone, C. J. (1971) Testing Hypotheses, in Introduction to Statistical Theory, pp 52-110, Houhgton Mifflin, New York.

21. Winzor, D. J., and Sawyer, W. H. (1995) Quantitative Characterization of Ligand Binding, Wiley-Liss, New York.

22. Golombek, A. P., and Hendrich, M. P. (2003) Quantitative analysis of dinuclear manganese(II) EPR spectra, J. Magn. Reson. 165, $33-48$.

23. D'Souza, V. M., Brown, R. S., Bennett, B., and Holz, R. C. (2005) Characterization of the active site and insight into the binding mode of the anti-angiogenesis agent fumagillin to the manganese(II)-loaded methionyl aminopeptidase from Escherichia coli, J. Biol. Inorg. Chem. 10, 41-50.

24. Sun, L., VanderSpek, J., and Murphy, J. R. (1998) Isolation and characterization of iron-independent positive dominant mutants of the diphtheria toxin repressor DtxR, Proc. Natl. Acad. Sci. U.S.A. 95, 14985-14990.

25. Manabe, Y. C., Hatem, C. L., Kesavan, A. K., Durack, J., and Murphy, J. R. (2005) Both Corynebacterium diphtheriae DtxR(E175K) and Mycobacterium tuberculosis IdeR(D177K) Are Dominant Positive Repressors of IdeR-Regulated Genes in $M$. tuberculosis, Infect. Immun. 73, 5988-5994.

26. Love, J. F., vanderSpek, J. C., Marin, V., Guerrero, L., Logan, T. M., and Murphy, J. R. (2004) Genetic and biophysical studies of diphtheria toxin repressor (DtxR) and the hyperactive mutant DtxR(E175K) support a multistep model of activation, Proc. Natl. Acad. Sci. U.S.A. 101, 2506-2511.

27. Qiu, X., Verlinde, C. L. M. J., Zhang, S., Schmitt, M. P., Holmes, R. K., and Hol, W. G. J. (1995) Three-dimensional structure of the diphtheria toxin repressor in complex with divalent cation corepressors, Structure 3, 87-100.

28. Chou, C. J., Wisedchaisri, G., Monfeli, R. R., Oram, D. M., Holmes, R. K., Hol, W. G. J., and Beeson, C. (2004) Functional studies of the Mycobacterium tuberculosis iron-dependent regulator, J. Biol. Chem. 279, 53554-53561.

29. Chen, C. S. Y., White, A., Love, J., Murphy, J. R., and Ringe, D. (2000) Methyl groups of thymine bases are important for nucleic acid recognition by DtxR, Biochemistry 39, 10397-10407.

30. D'Souza, V. M., Bennett, B., Copik, A. J., and Holz, R. C. (2000) Divalent metal binding properties of the methionyl aminopeptidase from Escherichia coli, Biochemistry 39, 3817-3826.

31. Zhang, L. B., Crossley, M. J., Dixon, N. E., Ellis, P. J., Fisher, M. L., King, G. F., Lilley, P. E., MacLachlan, D., Pace, R. J., and Freeman, H. C. (1998) Spectroscopic identification of a dinuclear metal centre in manganese(II)-activated aminopeptidase P from Escherichia coli: Implications for human prolidase, $J$. Biol. Inorg. Chem. 3, 470-483.

32. Antanaitis, B. C., Brown, R. D., Chasteen, N. D., Freedman, J. H., Koenig, S. H., Lilienthal, H. R., Peisach, J., and Brewer, C. F. (1987) Electron-Paramagnetic Resonance and MagneticSusceptibility Studies of Dimanganese Concanavalin-A: Evidence for Antiferromagnetic Exchange Coupling, Biochemistry 26, 7932-7937.

33. Reiter, T. A., Reiter, N. J., and Rusnak, F. (2002) $\mathrm{Mn}^{2+}$ is a native metal ion activator for bacteriophage lambda protein phosphatase, Biochemistry 41, 15404-15409.

34. Cammack, R., Chapman, A., Lu, W. P., Karagouni, A., and Kelly, D. P. (1989) Evidence That Protein-B of the Thiosulfate-Oxidizing System of Thiobacillus versutus Contains a Binuclear Manganese Cluster, FEBS Lett. 253, 239-243.

35. Reczkowski, R. S., and Ash, D. E. (1992) EPR Evidence for Binuclear Mn(Ii) Centers in Rat-Liver Arginase, J. Am. Chem. Soc. 114, 10992-10994. 
36. Chae, M. Y., Omburo, G. A., Lindahl, P. A., and Raushel, F. M. (1993) Antiferromagnetic Coupling in the Binuclear Metal Cluster of Manganese-Substituted Phosphotriesterase, J. Am. Chem. Soc. 115, 12173-12174.

37. Poyner, R. R., and Reed, G. H. (1992) Structure of the Bis Divalent-Cation Complex with Phosphonoacetohydroxamate at the Active Site of Enolase, Biochemistry 31, 7166-7173.

38. Khangulov, S. V., Pessiki, P. J., Barynin, V. V., Ash, D. E., and Dismukes, G. C. (1995) Determination of the Metal-Ion Separation and Energies of the 3 Lowest Electronic States of Dimanganese(II,II) Complexes and Enzymes: Catalase and Liver Arginase, Biochemistry 34, 2015-2025.

39. Spiering, M. M., Ringer, D., Murphy, J. R., and Marletta, M. A. (2003) Metal stoichiometry and functional studies of the diphtheria toxin repressor, Proc. Natl. Acad. Sci. U.S.A. 100, 3808-3813.

40. Lieser, S. A., Davis, T. C., Helmann, J. D., and Cohen, S. M. (2003) DNA-binding and oligomerization studies of the mangan-
ese(II) metalloregulatory protein MntR from Bacillus subtilis, Biochemistry 42, 12634-12642.

41. Cornish-Bowden, A. (1995) Inhibition and Activation of Enzymes, in Fundamentals of Enzyme Kinetics, pp 102, Portland Press, London.

42. Jakubovics, N. S., and Jenkinson, H. F. (2001) Out of the iron age: New insights into the critical role of manganese homeostasis in bacteria, Microbiology 147, 1709-1718.

43. Finney, L. A., and O'Halloran, T. V. (2003) Transition metal speciation in the cell: Insights from the chemistry of metal ion receptors, Science 300, 931-936.

44. Eisenstadt, E., Fisher, S., Der, C. L., and Silver, S. (1973) Manganese Transport in Bacillus subtilis W23 during Growth and Sporulation, J. Bacteriol. 113, 1363-1372.

BI052288G 